\title{
Survival of patients with Motor Neurone Disease (MND) following referral for nutritional assessment
}

\author{
T. Gledhill, C. Ormerod, V. Lekharaju, A. Smith and P. Bliss \\ University Hospital Aintree, Longmoor Lane, Liverpool L9 7AL, UK
}

Motor Neurone Disease (MND) is a neurodegenerative disease with a median survival of 4.32 years ${ }^{(1)}$. Poor nutritional status may reduce survival. There are no randomised controlled trials to indicate whether enteral feeding affords a survival benefit. However, a recent Cochrane review supports the recommendation by the American Academy of Neurology that a percutaneous endoscopic gastrostomy (PEG) should be considered when there is symptomatic dysphagia or accelerated weight $\operatorname{loss}^{(2)}$.

We reviewed the records of patients with MND referred for nutritional assessment and obtained data on the decision for gastrostomy, mode of insertion and survival outcomes.

Thirty patients were referred for nutritional assessment between July 2009 and February 2011. Of these, we were able to review 27 outcomes of patients using hospital computer records and dietetic reviews.

\begin{tabular}{|c|c|c|c|c|c|c|}
\hline $\begin{array}{l}\text { Age at } \\
\text { diagnosis } \\
\text { (years) }\end{array}$ & $\begin{array}{c}\text { Age at referral } \\
\text { (years) }\end{array}$ & $\begin{array}{c}\text { Time to referral } \\
\text { (years) }\end{array}$ & $\begin{array}{l}\text { BMI at referral } \\
\qquad\left(\mathrm{kg} / \mathrm{m}^{2}\right)\end{array}$ & $\begin{array}{l}\text { Documented } \\
\text { weight loss at } \\
\text { referral }\end{array}$ & $\%$ weight loss & $\begin{array}{c}\text { NIV at initial } \\
\text { review }\end{array}$ \\
\hline $\begin{array}{l}\text { Mean } 65.8 \\
\text { Median } 68.5\end{array}$ & $\begin{array}{l}\text { Mean } 66.8 \\
\text { Median } 71\end{array}$ & $\begin{array}{l}1 \\
\text { (range 0-7) }\end{array}$ & $\begin{array}{l}\text { Mean } 21 \\
\text { Median } 20.7\end{array}$ & $\begin{array}{l}72 \% \\
(16 / 22)\end{array}$ & $\begin{array}{l}19.4 \% \\
(7-36.7 \%)\end{array}$ & $\begin{array}{l}59.1 \% \\
(16 / 27)\end{array}$ \\
\hline
\end{tabular}

BMI = body mass index, NIV = non-invasive ventilation

At review, $81.4 \%$ (22/27) were offered gastrostomy tube placement, of these $66 \%$ accepted (15/22). Of the patients who were not felt appropriate for gastrostomy tube placement, 3 were gaining weight and 2 were too unwell. Ten were listed for a radiologically inserted gastrostomy (RIG) and 5 patients were listed for a PEG. All patients offered a PEG had normal overnight oximetry and did not require NIV (non-invasive ventilation). Successful tube placement of was achieved in all patients with the exception of 1 RIG. This patient subsequently underwent a surgical jejunostomy. 1 patient had pain and pneumoperitoneum post RIG and was managed conservatively.

\begin{tabular}{|c|c|c|c|c|c|c|c|}
\hline & Not offered & MDT decision & Patient declined & Too unwell & Gastrostomy & PEG & RIG \\
\hline Died during follow up & $50 \%(6 / 12)$ & $33.3 \%(1 / 3)$ & $42.8 \%(3 / 7)$ & $100 \%(2 / 2)$ & $46.7 \%(7 / 15)$ & $40 \%(2 / 5)$ & $50 \%(5 / 10)$ \\
\hline \multirow{2}{*}{$\begin{array}{l}\text { Mean survival post } \\
\text { procedure/decision not to } \\
\text { offer procedure (months) }\end{array}$} & 4.9 & 9.66 & 4.2 & 0.5 & 7.3 & 9 & 6.6 \\
\hline & & & & & & & \\
\hline 30 day mortality & $41.6 \%$ & $0 \%$ & $28.5 \%$ & $100 \%$ & $13 \%$ & $0 \%$ & $20 \%$ \\
\hline
\end{tabular}

The results show high short-term mortality in patients with MND referred to the nutrition team, reflecting advanced disease when nutritional status deteriorates. Although numbers are small, the mean survival of those undergoing PEG was higher compared to RIG. This may be due to difference in respiratory function between the 2 groups. The high 30 day mortality in patients not offered a gastrostomy may reflect accelerated deterioration without adequate nutrition or advanced disease at initial review.

1. Czaplinski A, et al. (2006) Slower disease progression and prolonged survival in contemporary patients with amyotrophic lateral sclerosis: is the natural history of amyotrophic lateral sclerosis changing? Arch Neurol 63(8), p. 1139-43.

2. Katzberg HD \& Benatar M Enteral tube feeding for amyotrophic lateral sclerosis/motor neuron disease. Cochrane Database Syst Rev 2011(1), p. CD004030. 